\title{
A DISCUSSÃO DA ABORDAGEM TEMÁTICA: ESTADO DO CONHECIMENTO NO ENSINO DE CIÊNCIAS ${ }^{1}$
}

\author{
Eril Medeiros da Fonseca* \\ Renata Hernandez Lindemann ${ }^{* *}$ \\ Leandro Duso $^{* * *}$
}

\begin{abstract}
Resumo: Este trabalho é um recorte de uma pesquisa de estado do conhecimento, no âmbito do Mestrado Acadêmico em Ensino da Universidade Federal do Pampa. Assume-se como objetivo perceber o enfoque dos referenciais teóricos adotados sobre a abordagem temática e as contribuições das pesquisas para o trabalho com temas no Ensino de Ciências. Adotou-se como critério o Qualis A1 pela Classificação de Periódicos Quadriênio 2013-2016 da CAPES. As buscas foram realizadas na plataforma Sucupira, selecionando a área de avaliação ensino e a classificação A1. Verificou-se 15 periódicos relacionados ao Ensino de Ciências, destes 05 nacionais, nos quais se selecionaram 04 artigos de pesquisa. Os trabalhos demonstraram discussões e propostas de implementação sobre o trabalho com temas com professores da Educação Básica, sinalizando alternativas de reconfigurações curriculares, baseadas na perspectiva freireana e/ou em elementos ligados ao movimento CTS. As pesquisas apontaram avanços, ações e práticas no campo da formação de professores, dos quais: possibilidade de repensar a organização curricular; superação do reducionismo metodológico e fragmentação do currículo; forma de relacionar temas de relevância social a conhecimentos científicos; envolvimento em um trabalho interdisciplinar. Sinaliza-se a importância de discussões sobre os objetivos e concepções de currículo, considerando a formação de professores como um campo profícuo de inovações, contribuindo para um constante (re)fazer de ações e reflexões. Nesse processo o papel do professor também é ressignificado, pois desconstrói a visão fragmentada de currículo e ensino.
\end{abstract}

Palavras-chave: Estado do conhecimento. Ensino de Ciências. Formação de professores. Abordagem temática.

\section{Introdução}

A valorização das contextualizações das escolas de Educação Básica constitui-se uma possibilidade de trabalho e organização didático-metodológica no Ensino de Ciências, que se opõem a uma abordagem individualista e reducionista da realidade. Rocha e Maestrelli (2016) discutem um cenário educativo que se materializa por um ensino conteudista, métodos autoritários, silenciamento das expressões dos alunos e conceitos distantes da realidade, em

\footnotetext{
${ }^{1}$ Este trabalho possui apoio da FAPERGS/CAPES

* Licenciado em Ciências da Natureza, mestrando em Ensino - Universidade Federal do Pampa (Unipampa).

** Doutora em Educação Científico-Tecnológica - Universidade Federal de Santa Catarina (Ufsc). Professora Adjunta da Unipampa.

**** Doutor em Educação Científico-Tecnológica e professor adjunto da Ufsc.

\# Tear: Revista de Educação Ciência e Tecnologia, Canoas, v.7, n.2, 2018. 
que o compromisso da ação pedagógica parece ser o currículo e não a aprendizagem do aluno, preocupando-se na carga conceitual e não no diálogo e sem questionar o porquê e para que se deva ministrar certos conteúdos.

Nesse sentido, no âmbito do Ensino de Ciências, a abordagem temática (AT) constituise uma perspectiva no processo de pesquisa/intervenção aliado ao ensino, viabilizando discussões mais amplas, não restritas apenas ao campo metodológico (AULER, 2003). Este mesmo autor menciona que a apropriação de conteúdos pela compreensão de temas coloca-se no intuito de instrumentalizar o estudante para atuação na sociedade contemporânea.

A AT aqui discutida está alicerçada nas concepções de Educação Libertadora de Freire (2016), que consiste na consideração do contexto social como ponto de partida da aprendizagem, balizados na dialogicidade e problematização. Nessa perspectiva os conceitos científicos são selecionados para atenderem a uma demanda de uma situação real e significativa que indica uma contradição da comunidade escolar - situação-limite - assim Freire propõe a organização curricular baseada em Temas Geradores (HALMENSCHLAGER, 2011).

Delizoicov, Angotti e Pernambuco (2011) inspirados no referencial de Paulo Freire e George Snyders discorrem sobre o planejamento didático-pedagógico com base em duas categorias de conhecimento: científico e de senso comum, em que este último é influenciado pelo conhecimento prévio do estudante. Tendo como ponto inicial os saberes em que o estudante já possui propõem um ensino pautado em temas, ou seja, a AT, definida pelos mesmos como uma perspectiva curricular em que a lógica estruturante são os temas, em que são selecionados os conteúdos de ensino.

Nesta perspectiva os temas constituem-se como objeto do conhecimento, em que o currículo escolar, a estruturação das atividades educativas, a seleção de conteúdos e a própria abordagem sistematizada em sala de aula rompem com o tradicional paradigma curricular, cujo princípio é a conceituação científica, ou seja, abordagem conceitual, em que a perspectiva curricular é estruturada pelos conceitos científicos, em que são selecionados os conteúdos de ensino (DELIZOICOV; ANGOTTI; PERNAMBUCO, 2011).

A estruturação da AT emerge do referencial freireano, que aborda os Temas Geradores a partir de uma investigação da realidade local. Assim, essas discussões consideram o fazer, pensar, agir, refletir, teoria e prática em relações que emergem de situações significativas, tanto individuais, como sociais e históricas, permitindo a discussão e interpretação da realidade, e a análise crítica do contexto, por meio do reconhecimento e intervenção no mesmo (DELIZOICOV; ANGOTTI; PERNAMBUCO, 2011; FREIRE, 2016). 


\section{\#tear}

Sendo assim, o presente trabalho trata de uma pesquisa de estado do conhecimento sobre a AT e suas articulações e implicações junto à área de Ensino. Assume-se como objetivo perceber o enfoque dos referenciais teóricos adotados sobre a AT e as contribuições das pesquisas para o trabalho com temas no Ensino de Ciências, sendo um recorte de uma pesquisa no âmbito do Mestrado Acadêmico em Ensino da Universidade Federal do Pampa.

\section{Abordagem Temática Freireana no Ensino de Ciências}

O Ensino de Ciências é definido segundo Chassot (2011), como uma linguagem para facilitar à leitura do mundo e também relacionada com o conceito de alfabetização científica (CHASSOT, 2011, p. 62): “[...] conjunto de conhecimentos que facilitariam aos homens e mulheres fazerem uma leitura do mundo em que vivem o que amplia o direito a escolha". Esta leitura possibilita uma apropriação de conceitos científicos, ampliando a dimensão de conhecimentos essenciais para a formação de uma postura em relação ao ambiente em que se vive.

Por isso defende-se, na Educação em Ciências, um ensino que proporcione uma visão e leitura de mundo pelo estudante de forma crítica e reflexiva (CHASSOT, 2011; DELIZOICOV; ANGOTTI; PERNAMBUCO, 2011; CACHAPUZ; PRAIA; JORGE, 2004; CACHAPUZ et al., 2011) de modo que este tenha capacidade de posicionar-se diante de problemáticas de cunho social, político, econômico e ambiental.

Desta forma apontamos a aproximação dessa discussão com as ideias de Freire (2013) quando o mesmo considera que para ensinar é necessário compreender que a Educação é uma forma de intervenção no mundo, o que contribui para pensarmos o papel social da Ciência, reforçando o compromisso da mesma na emancipação de sujeitos.

O sentido de considerar o que o educando já sabe é de que este seja capaz de realizar uma leitura de mundo e um reconhecimento crítico de sua realidade, identificando possíveis contradições a serem superadas, assim como Freire (1995, p. 83) considera um:

[...] trabalho pedagógico que, a partir do conhecimento que o aluno traz, [...], haja uma superação do mesmo, não no sentido de anular esse conhecimento ou de sobrepor um conhecimento ao outro. O que se propõe é que o conhecimento com o qual se trabalha na escola seja relevante e significativo para a formação do educando.

Consiste em um trabalho pedagógico que aprecie o conhecimento dos educandos, para problematizá-lo, identificando fragilidades e inconsistências, para então se abordar o conhecimento científico no sentido de entender melhor os fenômenos/situações ou a razão de 


\section{\#tear}

ser das mesmas (SILVA, 2015). Neste contexto os conceitos científicos passam a serem selecionados para suprirem necessidades no entendimento de situações reais e significativas, que expressam em certa medida uma situação-limite (HALMENSCHLAGER, 2011).

Assim Freire (2016) propõe a organização curricular a partir de Temas Geradores, que são obtidos pela Investigação Temática. Esse processo é sistematizado por Delizoicov (1982; 1991) a partir do terceiro capítulo da obra Pedagogia do Oprimido de Paulo Freire, e compreende as seguintes etapas: 1) Levantamento Preliminar: ocorre o estudo das condições da localidade, recolhendo informações e identificando possíveis situações-limite (contradições sociais vivenciadas pela comunidade); 2) Análise das Situações e Escolha das Codificações: as situações-limite são destacadas e preparam-se a sua codificação; 3) Diálogos Descodificadores: nesta etapa os problemas, representados pelos códigos, são discutidos e problematizados com a comunidade e obtém-se o Tema Gerador; 4) Redução Temática: cada educador seleciona possíveis conteúdos/conceitos a fim de potencializar o estudo do tema, com vistas à superação de determinada problemática; 5) Trabalho de Sala de Aula: constituise um processo educativo, intermediado pelo professor, com o programa estabelecido e os materiais a serem utilizados.

Para a realização desta última etapa Delizoicov e Angotti (1990) propõem os momentos pedagógicos. O primeiro momento denominado Problematização inicial é verificado as percepções dos estudantes sobre a situação significativa abordada. No segundo momento pedagógico, Organizações do conhecimento para dialogar com as questões levantadas pelos estudantes são selecionados os conhecimentos científicos relacionados a tais questões. Já no terceiro momento pedagógico, Aplicação do Conhecimento, o conhecimento científico é abordado de forma sistemática pelo estudante, para analisar, compreender e interpretar situações diferentes da inicial, no sentido de que o mesmo possa estabelecer relações com outras questões.

Quando estes momentos são desenvolvidos a partir de aspectos da vivência do estudante e de maneira dialógica contribuem para desenvolver no estudante o senso crítico e elevar seu nível de consciência (HALMENSCHLAGER, 2011). Para Freire (2016) a construção do conhecimento, visando à transformação deve ocorrer pela transição de um nível de consciência real para uma consciência máxima possível.

Assim, por meio do processo de Investigação Temática tanto docente quanto estudante apropria-se da realidade, reconhecem às contradições existentes no contexto, que se configuram segundo Freire (2016) como códigos a fim de serem desvelados. Além de propiciar uma maior aproximação entre professor-estudante e estudante-estudante. 


\section{\#tear}

Diante do exposto o trabalho a partir da Abordagem Temática Freireana (ATF), utilizando-se do processo de Investigação Temática ou de elementos do mesmo possibilitam articular um ensino contextualizado, de forma dialógica e problematizadora em sistemas de ensino. Estes elementos tencionam a discussão sobre (re)estruturação curricular, que é defendida e estudada por alguns autores da área de Ciências, a exemplo de Auler (2007a), Giacomini e Muenchen (2016), Muenchen e Auler (2007) e Halmenschlager (2010;2011).

\section{Delineamento das buscas}

As pesquisas de "estado do conhecimento" representam uma contribuição importante para o campo teórico de determinada área do conhecimento, pois possibilitam perceber o enfoque das temáticas que estão sendo desenvolvidas, bem como referenciais teóricos da investigação e contribuições das pesquisas para possíveis inovações e/ou mudanças.

Abordamos a denominação de estado do conhecimento ${ }^{2}$, por caracterizar-se como um estudo que aborda apenas um setor das publicações em relação ao tema pesquisado (ROMANOWSKI; ENS, 2006), neste caso periódicos nacionais e online de Qualis $\mathrm{A}^{3}{ }^{3}$ relacionados ao Ensino de Ciências.

Assim, os trabalhos selecionados obedeceram alguns critérios: artigos que apresentassem no título, resumo ou palavras-chaves o termo abordagem temática. Os periódicos selecionados para as buscas tiveram como critério o Qualis A1 de acordo com a Classificação de Periódicos Quadriênio 2013-2016 da CAPES. As buscas foram realizadas na plataforma Sucupira ${ }^{4}$, selecionando o quadriênio 2013-2016, a área de avaliação ensino e a classificação A1.

Após o levantamento dos periódicos e para melhor sistematização dos artigos encontrados, as informações como: palavras-chave da busca, título, ano de publicação, autores, instituição de ensino, nível e modalidade de ensino, região da instituição do primeiro autor, questão de pesquisa, objetivos da pesquisa, fundamentação teórica, metodologia da pesquisa e resultados encontrados foram compiladas em uma planilha do excel. Tais dados

\footnotetext{
${ }^{2}$ Vale ressaltar que, de acordo com um levantamento prévio, para definição dos termos "estado da arte" e "estado do conhecimento" não se encontrou aportes que distinguissem claramente as duas designações, pois alguns trabalhos abordam como sinônimos.

${ }^{3}$ É importante ressaltar que este trabalho caracteriza-se por uma busca inicial realizada apenas em periódicos nacionais e online de Qualis A1, devido à relevância dos mesmos para a pesquisa em Educação em Ciências, sendo que posteriormente as buscas serão aprofundadas em outros extratos.

${ }^{4}$ É uma ferramenta para coletar informações, realizar análises e avaliações, sendo a referência do Sistema Nacional de Pós-Graduação. Disponibiliza em tempo real informações, processos e procedimentos que a CAPES realiza em toda a comunidade acadêmica. O nome é em homenagem ao professor Newton Sucupira, autor do Parecer $n^{\circ} 977$ de 1965 , documento que conceituou e institucionalizou a pós-graduação brasileira (CAPES, 2014). Disponível em: https://goo.gl/ddrNpc.
} 
foram extraídos a partir da leitura dos resumos dos textos, e essa sistematização auxiliou na análise. Como forma de facilitar a apresentação dos materiais selecionados os periódicos nacionais foram identificados com a letra $\mathrm{P}$ seguido de um número, (P1, P2, P3, P4), da mesma forma os artigos foram representados como "Art" e respectivo numeral (Art1, Art2, Artn...).

\section{Análise e Discussões}

A busca na plataforma Sucupira indicou 145 periódicos de Qualis A1. Verificaram-se 15 periódicos relacionados ao Ensino de Ciências, destes 05 nacionais e online, em que se selecionaram 04 artigos de pesquisa. No quadro a seguir identificamos os periódicos, sua identificação e o ano em iniciaram suas publicações online.

Quadro 1 - Relação dos periódicos e ano de disponibilidade

\begin{tabular}{|l|c|c|}
\hline Periódico & Identificação & Disponível on-line desde \\
\hline Ciência \& Educação (online) & P1 & 1998 \\
\hline Educação em Revista (UFMG) & P2 & 2006 \\
\hline Educação em Revista (UNESP) & P3 & 2001 \\
\hline Ensaio. Pesquisa em Educação em Ciências & P4 & 1999 \\
\hline Revista Brasileira de Ensino de Física & P5 & 1979 \\
\hline
\end{tabular}

Em relação aos objetivos das pesquisas, é destacado no Art2.P1 discutir posicionamentos de professores da Educação de Jovens e Adultos (EJA) sobre a utilização de temas de relevância social nas aulas e identificar algumas dificuldades a serem superadas pelas instituições escolares. O Art3.P2 buscou analisar os avanços sobre intervenções curriculares pela AT na percepção de professores de EJA de uma escola pública. Da mesma forma a intenção do Art5.P4 foi discutir limites e possibilidades da AT, analisando as compreensões de professores da Educação Básica. E o Art6.P5 identificou elementos para o desenvolvimento de propostas escolares no Ensino de Ciências/Física através de visitas a centros escolares. No quadro a seguir demonstramos o principal enfoque dos artigos de pesquisa, selecionados para a análise.

Quadro 2 - Principal enfoque dos artigos

\begin{tabular}{|l|l|}
\hline Identificação & Enfoque \\
\hline Art2.P1 & Pesquisa sobre Formação de professores de Ciências \\
\hline Art3.P2 & Pesquisa sobre Formação de professores \\
\hline Art5.P4 & Pesquisa sobre Formação de professores de Física \\
\hline Art6.P5 & Pesquisa com implicações para o Ensino de Física \\
\hline \multicolumn{2}{|c|}{ Fonte: Os autores }
\end{tabular}


A partir do Quadro 2 e de uma leitura mais sistemática dos artigos foi possível perceber três trabalhos (Art2.P1, Art3.P1, Art5.P4) que abordam posicionamentos, compreensões e percepções de professores da Educação Básica quanto a utilização de temas e problemas de relevância social em sala de aula, bem como possibilidades de implementação, através de intervenções curriculares pautadas pela AT. O Art2.P1 e Art3.P2 possuem como sujeitos de pesquisa professores da EJA, levando em consideração a compreensão dos mesmos sobre a utilização de temas.

Já o Art6.P5 discutiu elementos, a partir da opinião de professores, a serem considerados na seleção e organização de conteúdos abordados nas visitas ao Laboratório Aberto de Física Nuclear, por estudantes do Ensino Médio. O mesmo considera que visitas dessa natureza contribuem para "[...] a construção de uma sociedade alfabetizada em ciência e tecnologia, capaz de refletir criticamente e atuar em situações vinculadas ao desenvolvimento científico-tecnológico" (WATANABE-CARAMELLO et al., 2010, p. 2). Percebe-se que o Art6.P5 buscou elementos em atividades de extensão que subsidiassem o desenvolvimento de propostas escolares no intuito de contribuir para uma sociedade cientificamente alfabetizada e com pesquisas que consideram tais aspectos. Em suma, os artigos Art2.P1, Art3.P2, Art5.P4 e Art6.P5, tratam de pesquisas relacionadas a formação de professores envolvendo elementos da AT.

Em relação à ATF as pesquisas analisadas apontam certa compreensão sobre esta perspectiva de trabalho por parte dos seus sujeitos de pesquisas, professores da Educação Básica, visto que realizaram cursos de formação continuada abordando a questão de temas em sala de aula. Nesta perspectiva, a formação de professores é apontada como uma alternativa para implementação desta abordagem, visto que mobiliza uma mudança na concepção de trabalho, em que os conteúdos passam a serem submetidos à compreensão dos temas e não ao contrário. Mobilizam também uma mudança na concepção de ensino e sobre o desenvolvimento da própria prática.

Sobre esta questão Strieder, Caramello e Gehlen (2010, p. 11) mencionam que para a efetiva implementação da proposta da AT é necessário “[...] uma formação dos professores em sintonia com propostas dessa natureza. Isto é, há necessidade do envolvimento destes professores em projetos que tem como propósito o desenvolvimento e implementação da Abordagem Temática [...]”. Neste sentido, o trabalho de Magoga et al. (2014), que analisou 35 trabalhos sobre práticas educativas baseadas na AT nas atas do Encontro Nacional de Pesquisa em Educação em Ciências (ENPEC), verificou que quase a metade dos trabalhos faziam menção direta ou indireta a necessidade de formação de professores. Desta forma, 
destaca-se a importância da formação de professores, em especial de caráter continuado/permanente, com vistas à discussão, implementação e reflexão sobre aspectos referentes à ATF.

Em certa medida percebeu-se possibilidades de implementação em razão de propostas de intervenção e investigação sobre práticas envolvendo elementos da ATF. Outro aspecto sinalizado que é pertinente para se trabalhar com temas, além da ATF é o trabalho a partir da perspectiva de temas de relevância social, inspirados em referenciais do movimento CiênciaTecnologia-Sociedade (CTS), ou os que articulam estes com pressupostos do educador Paulo Freire (AULER, 2007b; DELIZOICOV, 2008).

Estes aspectos pensados enquanto reconfiguração curricular ganham destaque em proporções gradativas em espaços de ensino como destacam Caramello, Strieder e Gehlen (2012, p. 206): “A discussão acerca do conteúdo programático da Educação Básica ser organizado por meio de temas parece ganhar, cada vez mais, espaço nas reflexões voltadas ao ensino".

Entende-se que o trabalho com temas requer uma dedicação, empenho e mudança na concepção de ensino, trabalho e currículo, além de uma equipe que possibilite pensar este trabalho de forma coletiva, por isso constitui-se enquanto processo de ação e reflexão. Neste sentido, Magoga et al. (2014) sinalizam ainda que durante as nove primeiras edições dos ENPECs houve um aumento no número de trabalhos sobre práticas educativas que abrangem a AT, indicando a disseminação desta perspectiva e a relevância de propostas e intervenções pautadas por temas.

Assim, os artigos aqui analisados são exemplos de propostas de implementação e de trabalho com professores da Educação Básica que apontam alternativas favoráveis a mudança e discussão de reconfigurações curriculares, baseadas tanto pela perspectiva freireana e/ou em elementos ligados ao movimento CTS, aspecto que também foi identificado por Magoga et al. (2015).

Percebeu-se que Auler (2002), Delizoicov, Angotti e Pernambuco (2011) e Freire (2016) são indicados como referenciais fortemente utilizados na discussão sobre AT, já que foram abordados por grande parte dos trabalhos (Art2.P1, Art3.P2, Art5.P4). Assim, os artigos dialogam seus referenciais teóricos entre autores que discutem elementos da AT, e alguns articulam aspectos relacionados à CTS, como o Art2.P1 e o Art3.P2 que são da mesma autora e estão balizados por aproximações entre os pressupostos de Paulo Freire e referenciais ligados ao movimento CTS. De forma análoga o Art6.P5 reflete a perspectiva dos temas com enfoque CTS, baseando-se em Santos e Schnetzler (2003), defendendo a potencialidade da 


\section{\#tear}

abordagem de temas para serem trabalhados no processo de ensino e aprendizagem, e ainda discussões entre CTS e o desenvolvimento de atitudes para a tomada de decisões. Já os Art2.P1, Art5.P4 e Art6.P5 abordaram a tese de Auler (2002), na qual o autor aponta relações entre a perspectiva freireana de Educação e o enfoque CTS como indicativo de articulação no Ensino de Ciências.

Diante dessas análises aponta-se que a AT no Ensino de Ciências caracteriza-se como uma possibilidade de (re)pensar a organização do currículo e suscitar uma mudança no formato do mesmo, de modo a contribuir também para o planejamento docente, o que perpassa a necessidade da mobilização de um trabalho coletivo, enquanto equipe articuladora de um ensino contextualizado.

Nesta perspectiva, o trabalho a partir de temas ganha sentido por provocar um fazer interdisciplinar, em que o conteúdo não se configura como um fim no processo de ensino e aprendizagem, mas um meio que possibilita responder questões de relevância sócio-política e ambiental, carregadas de significado mediante o contexto.

\section{Considerações acerca dos achados}

Os trabalhos demonstraram algumas aproximações acerca da ATF como a sinalização para a formação de professores e articulação de aspectos do contexto local e/ou realidade em processos de ensino e aprendizagem. As discussões sobre ATF enquanto proposta de intervenção e/ou possibilidade de reformulação curricular demandam um saber-fazer docente que pode ser viabilizado por um processo de formação, visto o próprio reconhecimento pelos autores dos trabalhos, em relação à ATF como mudança curricular e não restrito apenas ao campo metodológico.

Porém, além da formação de professores a abordagem de temas exige um trabalho coletivo e tempo de planejamento, que se constituem como desafios frente à estrutura hegemonicamente técnica/tradicional que os sistemas de ensino se inserem. Outra aproximação identificada que se relaciona tanto com a perspectiva de trabalho com temas como a ATF são as articulações com elementos do movimento CTS e aspectos de relevância social.

Neste sentido, aponta-se o papel da escola frente à reflexão da organização curricular, pois a mesma possui autonomia para discutir tais questões, sabendo que essa autonomia não se restringe apenas a estrutura e manutenção de recursos, pois os processos formativos de seus professores também perpassam sua autogestão. 
Sendo assim, identificou-se como uma possível lacuna nos trabalhos analisados justamente a ausência da consideração da gestão das instituições de ensino nas discussões sobre ATF como reconfiguração curricular. Assim, em que medida pode-se incluir a formação dos gestores nesse processo de reconstrução do currículo? A formação de professores seria o único elemento formativo a ser considerado no trabalho com a ATF?

Aponta-se também como fragilidade das produções a ausência das considerações discentes diante das propostas que envolvem a ATF, visto que as intervenções nesta perspectiva visam qualificar as práticas educativas no ensino e na aprendizagem. Apenas o Art5.P4 apontou a necessidade de envolver estudantes e professores em projetos vinculados a AT.

Convém destacar que mesmo a ATF sendo uma alternativa viável em processos de mudança curricular constitui-se um desafio uma vez que mobiliza discussões e mudanças de paradigma em relação a condições e carga horária de trabalho, disponibilidade docente, concepção de ensino, da profissão e da própria prática.

Por isso, convergentes com essas discussões a formação de professores aliadas a políticas públicas que garantam um trabalho coletivo/interdisciplinar, por meio de planejamentos e reuniões são alternativas favoráveis no trabalho com a ATF (MAGOGA et al., 2015).

Em síntese foi possível observar a partir dos trabalhos analisados anteriormente que todos (Art2.P1, Art3.P2, Art5.P4, Art6.P5) discutem princípios da AT em razão de diversos subsídios que contribuem para o Ensino, no sentido de qualificar as ações e práticas no campo da formação de professores, percebendo avanços e realizando apontamentos nas articulações do processo de implementação, dentre eles: possibilidade de repensar a organização curricular; superação do reducionismo metodológico e da fragmentação do currículo; forma de relacionar temas de relevância social a conhecimentos científicos; envolvimento em um trabalho interdisciplinar.

Sinalizam-se para a importância, na área de Ensino de Ciências, de algumas discussões sobre as concepções de currículo e, sobretudo os objetivos que se almejam no processo de estruturação deste. Tais discussões tornam-se possíveis na medida em se considera a dimensão da formação de professores como um campo profícuo de inovações, que possam contribuir para a área, a partir de um constante (re)fazer de ações e reflexões. Nesse processo o papel do professor também é ressignificado, na medida em que desconstrói a visão fragmentada de currículo e ensino. 


\title{
DISCUSSIONS OF THE THEMATIC APPROACH: THE STATE OF KNOWLEDGE IN SCIENCE TEACHING
}

\begin{abstract}
This study is nested within a larger research project on the state of knowledge, conducted within the Academic Masters in Education at the Universidade Federal do Pampa. The objective is to survey the focus of theoretical references on the thematic approaches and the contributions that these research studies have to make to working with themes in Science Teaching. The selection criterion was a Qualis A1 rating according to the 2013-2016 CAPES four-yearly classification of periodicals. Searches were run on the Sucupira platform, with the filters education and A1 classification. Fifteen periodicals were listed that deal with Science Teaching, five of which are Brazilian. A total of four research articles were selected for the study. These articles discussed implementation proposals involving thematic approaches for Elementary School teachers including suggestions for reformulation of curricula based on the Freirean perspective and/or on elements linked to Science-Technology-Society teaching. They reported on developments, activities, and practices in the field of teacher training including: the possibility of rethinking curricular organization; overcoming methodological reductionism and curricular fragmentation; means of relating socially relevant themes to scientific knowledge; and involvement in interdisciplinary work. The findings illustrate the importance of discussing the objectives and conceptions of curricula, considering teacher training as a fertile field for innovations, and contributing to a constant reworking of actions and reflections. As part of this process, the role of the teacher is also resignified, since it deconstructs the fragmented perspective on curricula and teaching.
\end{abstract}

Keywords: State of knowledge. Science teaching. Teacher training. Thematic approach.

\section{Referências}

AULER, D. Interações entre CTS no Contexto da Formação de Professores de Ciências. Tese (Doutorado) - Centro de Ciências da Educação, Universidade Federal de Santa Catarina, Florianópolis, 2002.

Alfabetização Científico-Tecnológica: um novo "paradigma"? ENSAIO : Pesquisa em Educação em Ciências, v. 5, n. 1, 2003.

. Enfoque Ciência-Tecnologia-Sociedade: pressupostos para o contexto brasileiro. Ciência \& Ensino, Piracicaba (SP), v. 1, n. especial, p. 1-20, 2007 a.

Articulação Entre Pressupostos do Educador Paulo Freire e do Movimento CTS: Novos Caminhos Para a Educação em Ciências. Contexto e Educação. v. 22. n.77; p.167188; Jan./Jun./2007b.

CACHAPUZ, A.; PRAIA, J.; JORGE, M. Da Educação em Ciência às orientações para o ensino das ciências: um repensar Epistemológico. Revista Ciência \& Educação, v. 10, n. 3, p. 363-381, 2004.

CACHAPUZ, A.; GIL-PEREZ, D.; CARVALHO, A. M. P. de; PRAIA, J.; VILCHES, A. A necessária renovação do ensino de ciências. 3. ed. São Paulo: Cortez, 2011. 


\section{\#tear}

CARAMELLO, G.W.; STRIEDER, R.B.; GEHLEN, S.T. Desafios e possibilidades para a abordagem de temas ambientais em aulas de Física. Revista Brasileira de Pesquisa em Educação em Ciências (RBPEC). v.12; n.1; p.205-222; Setembro/2012.

CHASSOT, A. Alfabetização Científica: questões e desafios para a educação. 5 ed. Ijuí: Unijuí, 2011.

DELIZOICOV, D. Concepção problematizadora do ensino de ciências na educação formal. Dissertação de mestrado. São Paulo: IFUSP/FEUSP, 1982.

Conhecimento, tensões e transições. 1991. Tese. (Doutorado em Educação) Faculdade de Educação, Universidade de São Paulo, São Paulo, 1991.

La Educación em Ciências y La Perspectiva de Paulo Freire. In: Alexandria Revista de Educação em Ciências e Tecnologia, v. 1, n. 2, Florianópolis, 2008.

.; ANGOTTI, J. A. Metodologia do Ensino de Ciências. São Paulo: Cortez, 1990.

; ANGOTTI, J. A.; PERNAMBUCO, M. M. Ensino de Ciências: Fundamentos e Métodos. São Paulo: Cortez, 2011.

FREIRE, P. Educação na Cidade. São Paulo: Cortez Editora. 1995.

Pedagogia da autonomia: saberes necessários à prática educativa. 9. ed. São Paulo: Paz e Terra, 2013.

Pedagogia do Oprimido. 60ª ed. Rio de Janeiro: Paz e Terra, 2016.

GIACOMINI, A.; MUENCHEN, C. Avanços alcançados por professores na implementação da abordagem temática em uma escola pública estadual do interior do RS. Educação em Revista, Belo Horizonte, v.32, n.03, p. 189-216, jul/set, 2016.

HALMENSCHLAGER, K. R. Abordagem Temática: Análise da Situação de Estudo no Ensino Médio da EFA. Florianópolis: PPGECT/UFSC, 2010. Dissertação, Universidade Federal de Santa Catarina, 2010.

Abordagem temática no Ensino de Ciências: Algumas possibilidades. Vivências, v, 7, n.13, p.10-21, Out, 2011.

MAGOGA, T.; CENTA, F. G,; SCHNEIDER, T. M.; MUENCHEN, C. Uma análise das práticas educativas baseadas na abordagem temática nas atas dos ENPECs. In: III Seminário Internacional de Educação em Ciências, 2014, Rio Grande/RS. Anais... Rio Grande/RS: Universidade Federal de Rio Grande, 2014. Disponível em: <http://www.casaleiria.com.br/sintec3/sintec3.htm> Acesso em: 03 jan. 2018.

MAGOGA, T.; SCHNEIDER, T. M.; CENTA, F. G.; MUENCHEN, C.A escolha dos temas em práticas educativas baseadas na abordagem temática. Vivências: Revista Eletrônica de Extensão da URI, Erechim/RS, v. 11, n. 21, p. 10-22, out. 2015.

MUENCHEN, C.; AULER, D. Configurações curriculares mediante o enfoque CTS: Desafios a serem enfrentados na educação de jovens e adultos. Ciência \& Educação, v. 13, n. 3, p. 421-434, 2007. 
ROCHA, A. L. F. da; MAESTRELLI, S. R. P. A formação de professores crítico-reflexiva: pressupostos necessários ao desvelar colaborativo da prática docente. In: PAULINO, V.; BARBOSA, A. T. (Org.). Língua, Ciência e Formação de professores em Timor-Leste. 1ed. Díli: Unidade de Produção e Disseminação do Conhecimento/Programa de Pósgraduação e Pesquisa da UNTL, v. 01, p. 93-102, 2016.

ROMANOWSKI, J. P.; ENS, R. T. As pesquisas denominadas do tipo "estado da arte" em educação. Diálogo Educacional, Curitiba, v. 6, n. 19, p. 37-50, set./dez. 2006.

SANTOS, W.L.P.; SCHNETZLER, R.P. Educação em Química: Compromisso com a cidadania. Editora da UNIJUI, Ijuí, 2003.

SILVA, R. M. A abordagem temática freireana na formação de professores de ciências sob a optica da teoria da atividade. Dissertação. Programa de Pós-graduação em Educação Científica e Formação de Professores. Universidade Estadual do Sudoeste da Bahia. 2015.

SILVA, R. M. A abordagem temática freireana na formação de professores de ciências sob a optica da teoria da atividade. Dissertação. Programa de Pós-graduação em Educação Científica e Formação de Professores. Universidade Estadual do Sudoeste da Bahia. 2015.

STRIEDER, R. B.; CARAMELLO, G. W; GEHLEN, S. T. Abordagem de temas no ensino médio: compreensões de professores de física. In: Atas do XII Encontro de Pesquisa em Ensino de Física. Águas de Lindóia/SP, 2010.

WATANABE-CARAMELLO, G.; STRIEDER, R. B.; WATANABE, G.; MUNHOZ, M. G. Articulação Centro de Pesquisa - Escola Básica: Contribuições para a Alfabetização Científica e Tecnológica. Revista Brasileira de Ensino de Física, v. 32, n. 3, 2010. 